\title{
Neoadjuvant chemotherapy with or without neoadjuvant radiotherapy compared with neoadjuvant chemoradiotherapy for esophageal cancer
}

\author{
Yan Zheng ${ }^{1 \#}$, Xianben Liu ${ }^{1}$, Ruixiang Zhang ${ }^{1}$, Zongfei Wang ${ }^{1}$, Haibo Sun ${ }^{1}$, Jianjun Qin ${ }^{1,2}$, Shilei Liu ${ }^{1}$, \\ Yin $\mathrm{Li}^{1,2 \#}$ \\ ${ }^{1}$ Department of Thoracic Surgery, The Affiliated Cancer Hospital of Zhengzhou University, Henan Cancer Hospital, Zhengzhou 450008, China; \\ ${ }^{2}$ Department of Thoracic Surgery, National Cancer Center/National Clinical Research Center for Cancer/Cancer Hospital, Chinese Academy of \\ Medical Sciences and Peking Union Medical College, Beijing 100021, China \\ Contributions: (I) Conception and design: Y Li, Y Zheng; (II) Administrative support: Y Li, Y Zheng; (III) Provision of study materials or patients: All \\ authors; (IV) Collection and assembly of data: All authors; (V) Data analysis and interpretation: All authors; (VI) Manuscript writing: All authors; (VII) \\ Final approval of manuscript: All authors. \\ "These authors contributed equally to this work. \\ Correspondence to: Yin Li, MD, PhD. Department of Thoracic Surgery, National Cancer Center/National Clinical Research Center for Cancer/ \\ Cancer Hospital, Chinese Academy of Medical Sciences and Peking Union Medical College, Beijing 100021, China. Email: liyin0825@hotmail.com.
}

Background: Although it was controversial for treating locally advanced resectable esophageal squamous cell carcinoma (ESCC), neoadjuvant chemoradiotherapy (NACR) was more widely accepted rather than neoadjuvant chemotherapy (NAC) worldwide. With the development of paclitaxel, a high response rate to NAC was reported in many studies. Our hypothesis is that lots of patients could get a response from NAC alone and avoid unnecessary NACR. Those who had no response from NAC could still response from the followed radiotherapy. We attempted to circumvent the controversy over the use of NAC, NACR and made a combined version, $\mathrm{NAC} \pm$ neoadjuvant radiotherapy (NAR).

Methods: The retrospective study compared NAC \pm NAR with NACR between June 30, 2015 and October 31, 2016. Sixty consecutive borderline resectable ESCC were included: thirty-one in NAC \pm NAR group and 29 in NACR group. The toxicities, response rates, operative data, complications, length of stay, and overall survival (OS) rates were evaluated.

Results: The response rate to NAC \pm NAR was $93.5 \%$; to NACR was $86.2 \%$. There was no grade $3-4$ non-hematologic adverse events after NAC \pm NAR, but three in the NACR group. Arrhythmias $(6.5 \% v s$. $37.9 \%, \mathrm{P}=0.003)$, pneumonitis (25.8\% vs. $51.7 \%, \mathrm{P}=0.039)$ and anastomotic leakage $(0 \%$ vs. $13.8 \%, \mathrm{P}=0.049)$ were more likely in NACR group. Postoperative hospitalization stays were significantly prolonged in the NACR (9 vs. $16 \mathrm{~d}, \mathrm{P}<0.001$ ). A point estimate of the 2 -year OS rate of the NAC \pm NAR group was $84.0 \%$, the NACR group $80.7 \%(\mathrm{P}=0.410)$.

Conclusions: Compared with NACR, the NAC \pm NACR provided the same survival benefits but low post operation complication rate. In the future, it might be a choice for locally advanced ESCC.

Keywords: Neoadjuvant chemotherapy (NAC); neoadjuvant chemoradiotherapy (NACR); esophageal cancer

Submitted Sep 20, 2017. Accepted for publication Jul 18, 2018.

doi: $10.21037 /$ jtd.2018.07.124

View this article at: http://dx.doi.org/10.21037/jtd.2018.07.124 


\section{Introduction}

The standard of care for locally advanced resectable esophageal squamous cell carcinoma (ESCC) is neoadjuvant chemotherapy (NAC) in Japan $(1,2)$ and neoadjuvant chemoradiotherapy (NACR) in western countries (3). More evidence available is supporting the use of NACR worldwide, especially for locally advanced disease (4). Even in Japan, NACR is recommended in patients whose disease is suspected to be borderline resectable (5). However, the side effects of NACR cannot be ignored. In the Francophone de Cance'rologie Digestive 9901 (FFCD 9901) trials, the in-hospital postoperative mortality of NACR group was $11.1 \%$ (6). Surgical procedures following NACR were challenging, reflected in prolonged surgical times and blood loss. Also concerning was an increase in major postoperative complications (7).

Docetaxel achieved significantly longer survival in SCC of the head and neck (8) and locally advanced ESCC (9). A phase II study also suggested that preoperative docetaxel, cisplatin and fluorouracil (DCF) was well tolerated in ESCC (10). The regimen exhibited a response rate of $60.0 \%$ with no treatment-related deaths (10). In our retrospective data, paclitaxel + cis-platinum (TP) achieved an overall clinical response rate of $77.1 \%$. The pathological complete response (pCR) rate was $20.5 \%$, in contrast to $29 \%$ for similar patients receiving chemoradiotherapy alone reported by CROSS trial (3). These data suggested that, in addition to NACR, nowadays many patients could achieve a response only by chemotherapy.

Therefore, we designed NAC with or without NAR $(\mathrm{NAC} \pm \mathrm{NAR})$ combined treatment to avoid unnecessary NACR. The curative surgery was performed in patients who responded to NAC. Otherwise, NAR was employed.

We hypothesized that NAC \pm NAR compared with NACR combined treatment mode could significantly reduced the postoperative complications and postoperative hospital stays. Furthermore, they might have the same survival benefits.

\section{Methods}

\section{Inclusion criteria}

The project was approved by the Review Board and Ethics Committee of Henan Cancer Hospital $(\mathrm{HCH}) /$ The affiliated Cancer Hospital of Zhengzhou University. The ethical approval number is 2018122. At our institution, we have occasionally seen patients with locally advanced
ESCC that the longest diameter of transverse section of the tumor was longer than $3.3 \mathrm{~cm}$ and was suspected of uneasy surgery by computed tomography (CT) scan, however which was diagnosed as T3 disease by electronic ultrasonic esophagoscopy (EUS), bronchofiberscope, chest magnetic resonance imaging (MRI), and other preoperative tests. We refer to these cases as borderline-resectable cT3 cancer. We retrospectively collected the data of patients with borderlineresectable cT3 ESCC who received NAC \pm NAR and surgery in the First Ward Thoracic Surgery Department of HCH between June 30, 2015 and October 31, 2016. The control group was the patients with borderline-resectable T3 ESCC who received NACR and surgery in our department during the same time. The inclusion criteria were: clinical stage borderline-resectable T3N0-1M0 according to the 2012 TNM classification; aged 17 to 80 years; esophagectomy was done through right thoracic cavity; with sufficient bone marrow function; and without any contraindications due to conditions of the liver, kidneys, heart, or lungs.

\section{Treatment profile}

Figure 1 shown the clinical pathways of the NAC \pm NAR and NACR routes. In NAC \pm NAR group, therapy was started with NAC. According to the Response Evaluation Criteria in Solid Tumors (RECIST) version 1.1 (11), if patients achieved a response, partial response (PR)/complete response (CR), we proceeded to surgery. If the patients were instead found to have $\mathrm{SD} /$ progressive disease (PD), we defined it as no response and suggested the patients receive 30-45 Gy radiotherapy. After NAR, if the patients achieved response (PR/CR), surgery was employed. If the patients achieved no response (SD/PD), we suggested them to receive definitive radiotherapy or surgery. Reevaluation after all of these treatments, surgeries were accessed, and further treatments or palliative therapy were provided as necessary.

\section{$N A C \pm N A R$}

The NAC included two cycles of TP, consisting of paclitaxel at a dose of $175 \mathrm{mg} / \mathrm{m}^{2}$ and cisplatin at $75 \mathrm{mg} / \mathrm{m}^{2}$ by continuous infusion. No response patients would receive 35-40 Gy radiotherapy.

\section{NACR}

The concurrent NACR was performed with $6 \mathrm{MV}$ photons 


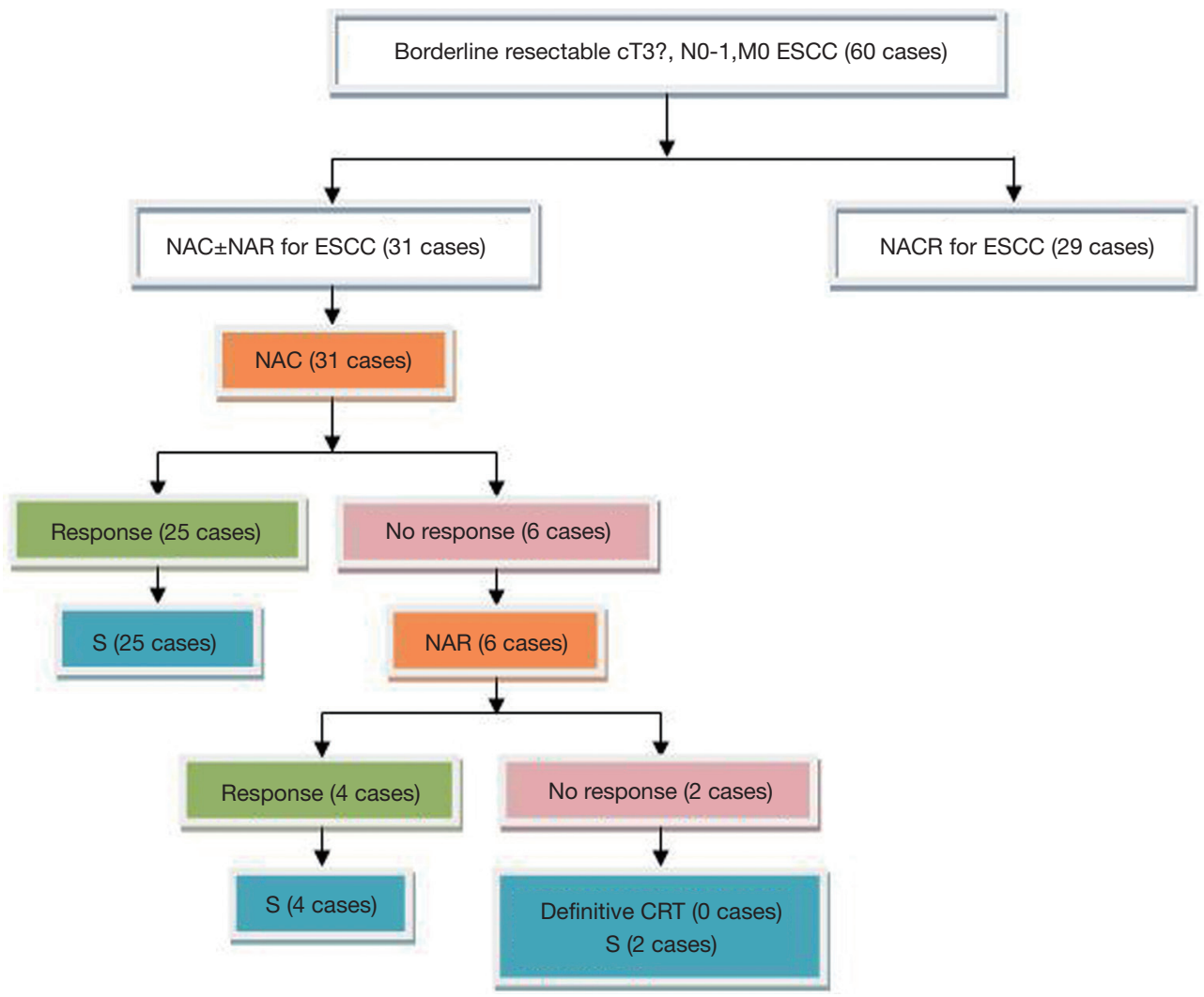

Figure 1 Flow diagram of inclusion of NAC \pm NAR combined treatment mode and NACR for Borderline Resectable cT3? N0-1, M0 ESCC from June 30, 2015 to October 31, 2016. NAC \pm NAR group was started with NAC. If patients achieved a response, partial response (PR)/complete response (CR), we proceeded to surgery. If the patients got no response, stable disease (SD)/progressive disease (PD), we recommended the patients receive 30-45 Gy radiotherapy. After NAR, if the patients achieved response, surgery was employed. If no response, definitive radiotherapy or surgery would be suggested. There were 60 patients included into the retrospective study. Thirty-one patients were treated with the NAC \pm NAR, and 29 patients commenced the NACR. In NAC \pm NAR group, after NAC, six patients were evaluated as SD. These patients received the followed NAR. Four of them got clinical PR, and 2 of them got SD. All of the two group patients achieved R0 resection.

to a total dose of 35-40 Gy over less than 5 weeks. If the patients could not undergo surgery, the radiotherapy was performed to a total dose of 60-65 Gy. During the course of NACR, docetaxel + cisplatin (DP) chemotherapy was administered with radiotherapy. Two cycles of DP consisted of docetaxel at $50 \mathrm{mg} / \mathrm{m}^{2}$ and cisplatin at $60 \mathrm{mg} / \mathrm{m}^{2}$.

\section{Surgical procedure}

Right thoracotomy esophagectomy and regional lymphadenectomy was performed. Thoracolaparoscopic esophagectomy was accepted. Transhiatal esophagectomy and left thoracotomy was excluded. Total two-field lymphadenectomy was adopted. The left recurrent laryngeal nerve, right recurrent laryngeal nerve, paraesophageal, paratracheal, subcarinal, supradiaphragmatic, posterior mediastinal lymph nodes, celiac, left gastric artery, common hepatic artery and splenic artery lymph nodes were all defined as the regional lymph nodes (12). Li's anastomosis (13) and gastric conduit were used for all patients.

\section{Clinical and pathological tumor response}

The esophagography, EUS, contrast-enhanced thoracic CT scan, abdominal echography, brain MRI, cervical color ultrasound and emission computed tomography (ECT) were 
Table 1 Characteristics and general data of patients with ESCC

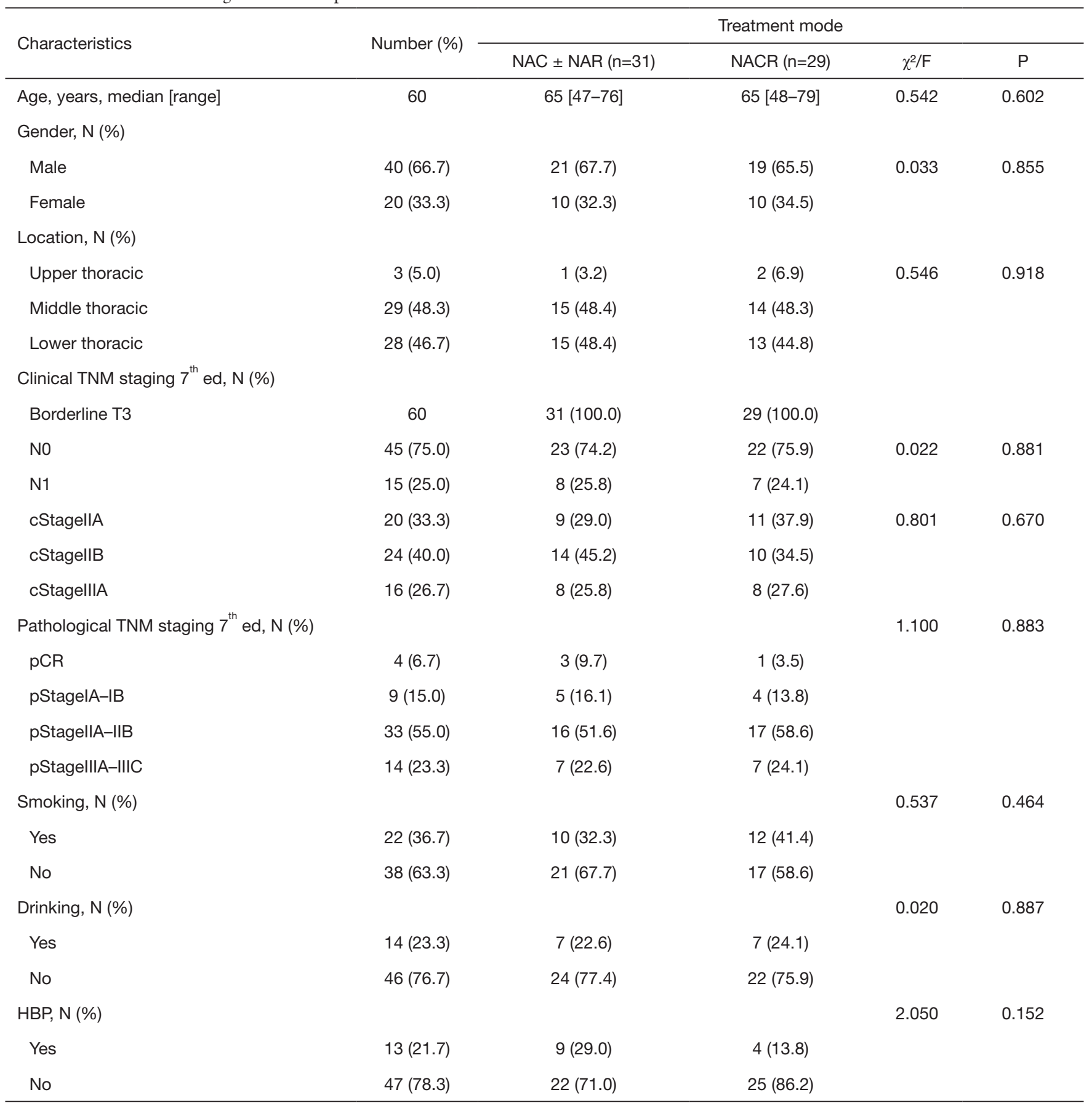

ESCC, esophagus squamous cell carcinoma; NAC \pm NAR, neoadjuvant chemotherapy with or without neoadjuvant radiotherapy; NACR, neoadjuvant chemoradiotherapy; TNM, tumor/node/metastasis; c, clinical; pCR, pathological complete response; HBP, high blood pressure.

essential pre-treatment examinations. PET/CT was used instead of abdominal echography, cervical color ultrasound and ECT if the patient had good financial circumstances.
The common Terminology Criteria for Adverse Events (CTCAE) Version 3.0 was used to assess the adverse events of NAC and NAR (14). The evaluation of the clinical tumor 
Table 2 Response rate to pre-surgery treatment

\begin{tabular}{lcc}
\hline Variable & $\mathrm{N}(\%)$ & $\%(95 \% \mathrm{Cl})$ \\
\hline Response after NAC (N=31) & $25(80.6)$ & $74.2-97.8$ \\
cCR + cPR & $2(6.5)$ & $0-14.3$ \\
pCR & & \\
Response after NAC \pm NAR (N=31) & $29(93.5)$ & $85.7-100$ \\
cCR + cPR & $3(9.7)$ & $0-19.5$ \\
pCR & & \\
Response after NACR (N=29) & $25(86.2)$ & $74.4-98$ \\
cCR + cPR & $1(3.4)$ & $0-9.4$ \\
pCR &
\end{tabular}

$\mathrm{N}$, number; $\mathrm{Cl}$, confidence interval; NAC $\pm \mathrm{NAR}$, neoadjuvant chemotherapy with or without neoadjuvant radiotherapy; cCR, clinical complete response; cPR, clinical partial response; $\mathrm{pCR}$, pathological complete response; NACR, neoadjuvant chemotherapy.

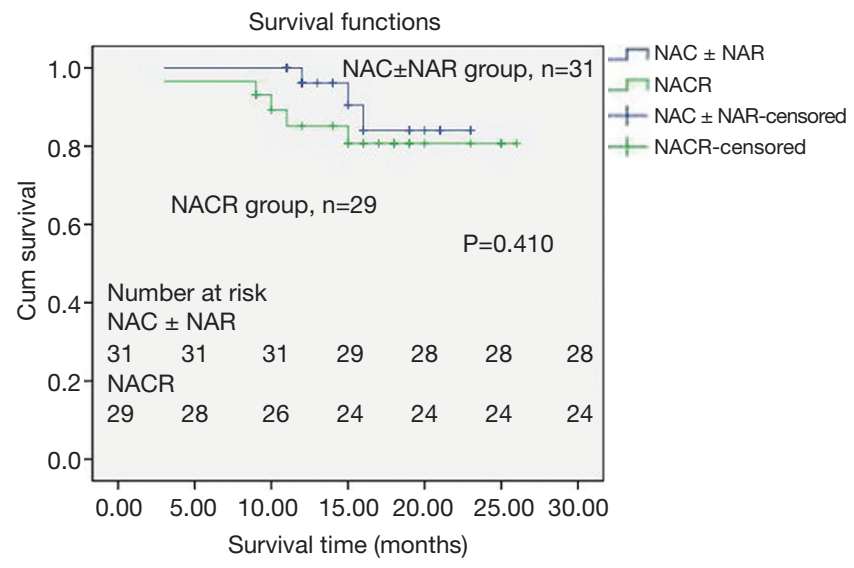

Figure 2 Kaplan-Meier overall survival analysis of ESCC patients in NAC \pm NAR and NACR groups $(n=60)$. The survival rates of patients in the NAC \pm NAR group and NACR group were without significant difference (log-rank test, $\mathrm{P}=0.410$ ). ESCC, esophagus squamous cell carcinoma; NAC, neoadjuvant chemotherapy; NAR, neoadjuvant radiotherapy; NACR, neoadjuvant chemotherapy.

responses were conducted by the RECIST 1.1 (11). No evidence of viable cancer cells was defined as pCR (15).

\section{Follow-up}

A research nurse contacted all patients by phone and ensured that each patient would be followed at outpatient clinics. The surveillance examinations included chest CT scans and abdominal, cervical echography routinely. The clinical and laboratory examinations were repeated every 3 months for first 3 years, every 6 months for the next 2 years. The end point was defined as death or being lost to follow-up.

\section{Statistical analysis}

The statistical analyses were conducted using SPSS 17.0 software for Windows (SPSS Inc., Chicago, IL, USA). Statistical significance was defined as a two-sided $\mathrm{P}$ value of 0.05. The pretreatment data were compared using a MannWhitney $U$ test and a Chi-square test for qualitative data and the student's $t$-test for all quantitative data. KaplanMeier curves and the log-rank test were used for statistical analysis of overall survival (OS). The OS was defined as the first date from the NAC/NACR to the tumor recurrence or most recent follow-up.

\section{Results \\ Patient characteristics}

There were 60 patients included into the retrospective study. Thirty-one patients were treated with the NAC \pm NAR, and 29 patients commenced the NACR. Baseline characteristics of all 60 patients are summarized in Table 1 . Most patients were male $(66.7 \%)$, and the median age was 65 years old (range, 47-79 years old). There were no significant differences between the two groups.

\section{Efficacy outcomes}

The response rate was summarized in Table 2. The overall response rate of $\mathrm{NAC} \pm \mathrm{NAR}$ was $93.5 \%$. After NAC, six patients were evaluated as SD. These patients received the followed NAR. Four of them got clinical PR, and 2 of them got SD. One of the clinical PR patients was evaluated pCR after surgery. All of the two group patients achieved R0 resection.

\section{Survival}

The median follow-up period in all patients surviving without tumor progression was 15.5 months (range, 3-26 months). The median OS for all patients was not reached. A point estimate of the 2-year OS rate of the NAC \pm NAR group 
Table 3 Side effects of neoadjuvant therapy

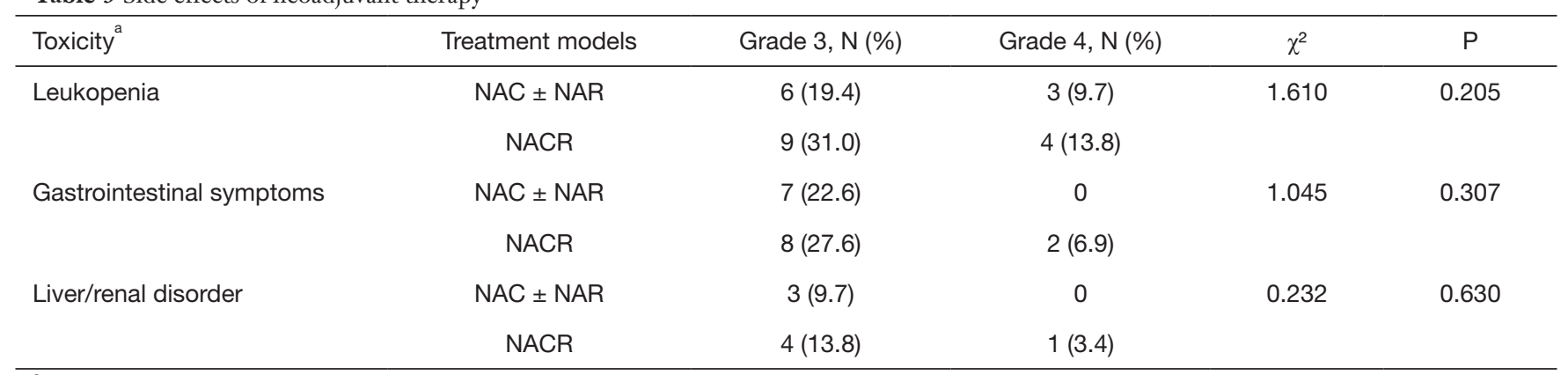

${ }^{a}$, Common Terminology Criteria for Adverse Events Version 3.0; N, number; NAC \pm NAR, neoadjuvant chemotherapy with or without neoadjuvant radiotherapy; NACR, neoadjuvant chemoradiotherapy; $\chi 2$ and $P$, the data from grade $3+$ grade 4 patients.

Table 4 Operation data for patients of NAC \pm NAR and NACR

\begin{tabular}{|c|c|c|c|}
\hline Variable & $N A C \pm N A R(N=31)$ & NACR $(\mathrm{N}=29)$ & $P$ \\
\hline Operation time (minutes), mean \pm SD & $237.26 \pm 73.369$ & $268.38 \pm 53.189$ & $0.024^{a}$ \\
\hline $\mathrm{EBL}(\mathrm{mL})$, median [range] & $100[50-290]$ & $200[50-2,000]$ & $<0.001^{a}$ \\
\hline Total nodes collected, median [range] & $29[10-51]$ & $22[10-44]$ & $0.007^{\mathrm{a}}$ \\
\hline Pathologically positive lymph nodes, median [range] & $0[0-11]$ & $0[0-15]$ & 0.978 \\
\hline Hospital mortality (\%) & 0 & 0 & NA \\
\hline Complication, N (\%) & $10(32.3)$ & $20(69.0)$ & $0.004^{a}$ \\
\hline Pneumonia & $8(25.8)$ & $15(51.7)$ & $0.039^{\mathrm{a}}$ \\
\hline ARDS & $3(9.7)$ & $5(17.2)$ & 0.630 \\
\hline
\end{tabular}

a , statistically significant $(\mathrm{P}<0.05)$. NAC \pm NAR, neoadjuvant chemotherapy with or without neoadjuvant radiotherapy; NACR, neoadjuvant chemoradiotherapy; N, number; SD, standard deviation; EBL, estimated blood loss; NA, not applicable; ARDS, acute respiratory distress syndrome.

was $84.0 \%$, whereas $80.7 \%$ in the NACR group (Figure 2). The OS of NAC \pm NAR and NACR were not significantly different (mean OS time: $21.672 \pm 0.710,95 \%$ CI, 20.28123.063 vs. $22.899 \pm 1.274$ months, $95 \%$ CI, 20.402-25.395; $\mathrm{P}=0.410)$.

\section{Adverse events}

The overall toxicities during treatment were listed in Table 3. The major toxicities were leukopenia and gastrointestinal symptoms. There were three in the NAC \pm NAR group (9.7\%) and seven in the NACR group (24.1\%) got adverse events of grade four. There was no significant difference between two groups. Surgical data for both groups are shown in Table 4. There was longer surgical time (mean, 237.26 73.369 vs. $268.38 \pm 53.189 \mathrm{~min}, \mathrm{P}=0.024)$, more blood loss (median, 100 vs. $200 \mathrm{~mL}, \mathrm{P}<0.001$ ) and longer postoperative stays (median, 9 vs. $16 \mathrm{~d}, \mathrm{P}<0.001$ ) in the NACR group. The total complication rates $(32.3 \%$ vs. $69 \%$, $\mathrm{P}=0.004)$, the rates of arrhythmia (6.5\% vs. $37.9 \%, \mathrm{P}=0.003)$ and pneumonitis $(25.8 \%$ vs. $51.7 \%, \mathrm{P}=0.039)$ were higher in the NACR group. The anastomotic leakage only developed in NACR patients ( $0 \%$ vs. $13.8 \%, \mathrm{P}=0.049)$. There are no treatment related deaths.

\section{Discussion}

This retrospective study was designed to compare the 
long-term and short-term outcomes of a new combined mode NAC \pm NRC with the standard NACR for ESCC. Finally we demonstrated NAC \pm NRC and NACR may have same 2-year OS rates. We also demonstrated NAC \pm NRC had significantly better surgical data, post operation complication rates, as compared with the standard NACR for ESCC.

The most controversial part of locally advanced resectable ESCC is whether to use NAC or NACR. More evidence supports the survival benefit of NACR compared with surgery alone (4). There were 2 clinical trials compared NAC and NACR for EC. They all got negative results, one of the trial got $\mathrm{P}$ value of 0.37 (16), the other $\mathrm{P}=0.07$ (17). Two meta analysis which focus on this topic also got a negative result $\mathrm{P}=0.07(4,18)$. A study reported that the addition of radiotherapy to NAC resulted in higher $\mathrm{pCR}$ rate, $\mathrm{R} 0$ resection rate, and a lower frequency of lymph-node metastases, however contributed nothing significant to survival (19). The difference of survival benefits between NAC and NACR may not be significant. As the high response rate of NACR for ESCC was observed, Japan started to do NExT Study (20) and Qun wang has launched to do NACR versus NAC for ESCC in China (21). Although they all expected a better survival results of NACR, the conclusion is still unclear. In this study, the NAC had become an induction chemotherapy for radiotherapy in no response patient. The induction chemotherapy prior to NACR also got a comparable survival benefit with NACR for ESCC (22). NAC \pm NAR was increased the response rate of NAC alone and comparable data of NACR. These reasons may contribute to our survival data. The NAC \pm NAR and NACR achieved a same survival benefits of 2 -year OS rate of $84 \%$ and $80.7 \%(\mathrm{P}=0.025)$. Furthermore, the 2 -year survival rate in the NAC \pm NAR group was higher than that in the group of standard-dose cisplatin, 5-Fu-radiotherapy in the CROSS trial (3). These data suggested that NAC \pm NAR might be a sufficiently powerful combine model that results in a high rate of response and 2-year OS.

Another concern is the side-effects and safety of preoperation treatment. The treatment related deaths cannot be ignored in the FFCD9901 trial (6). The side effects of neoadjuvant therapy are shown in Table 3. Three (9.7\%) NAC+NAR patients had grade 4 leukopenia. Four (13.8\%) NACR patients also developed grade 4 leukopenia. The side effects in two groups were quite acceptable. Compared with the data reported in other studies (23), it appears that the number of side effects in our study was quite low. In this study, we used paclitaxel $87.5 \mathrm{mg} / \mathrm{m}^{2}$, $\mathrm{d} 1, \mathrm{~d} 8$ and cisplatin $25 \mathrm{mg} / \mathrm{m}^{2}, \mathrm{~d} 2-\mathrm{d} 4$ every 3 weeks for 2 cycles. The weekly paclitaxel and the divided cisplatin could dramatically reduce the toxicity of $\mathrm{TP}$, which might be the reason why we achieved such minimal side effects. The surgical data for patients were listed in Table 4. The operation procedure for NACR was challenging, the surgical time and blood loss were statistically different. Postoperative pneumonia was more likely in NACR patients $(\mathrm{P}=0.039)$. Similar to FFCD9901, the lungs and heart were the main organs injured after NACR for ESCC (6). The results were also consistent with ESCC subgroup metaanalysis of Kumagai et al. (7). They suspected the ESCC usually had a long history of smoking and alcohol abuse which may be harmful to heart and lung (7). The number of postoperative days in the hospital was significantly prolonged in NACR group.

Additionally, ESCC is more common in developing regions worldwide $(24,25)$. Even within China, ESCC occurs more frequently in poor areas (26). It is clear that NAC has low side effects, acceptable, affordable, and therefore may easily be promoted (7). In another our retrospective study, we could achieve a 20.5\% PCR rate and a $77.1 \%$ response rate in ESCC by NAC alone; the data was promising. We learned that many patients could achieve enough response from NAC only. They do not need NACR for surgery. Why not combine NAC and NACR together? Since June of 2016 in our hospital, we have explored the $\mathrm{NAC} \pm \mathrm{NAR}$ model.

In our study, the NAC-SD patients could still attain high response rates from the followed NAR treatment. The NAR was not too late for the NAC SD patients (response rate $66.7 \%$ ). The combined NAC \pm NAR group achieved a highest response rate $(93.5 \%)$ than the NACR group $(86.2 \%)$. The clinical response rate of two-cycle CF in 9907 patients was $38 \%$ (1). The clinical response rate of two-cycle DCF was $64.3 \%$ (10). In our retrospective study of NAC for ESCC, the clinical response rate of two cycle TP was $77.1 \%$. The pCT of NACR in CROSS study was $29 \%, 33.3 \%$ in FFCD9901 (6). All the data shown above demonstrate that, with the development of a new chemotherapy regimen paclitaxel, more patients could obtain enough response from NAC for surgery. They did not need additional radiotherapy for local control. In our retrospective study, TP not only achieved a high response rate but also had low toxicity.

There were some limitations in this study that need to be acknowledged. One of the major concerns was that it 
was a retrospective study. There could have been a selection bias especially for response rates of neoadjuvant treatment. Secondly, this is a single institution study based on a small number of patients and a short observation period. Thirdly, for advanced esophageal cancer, the standard preoperation treatment in our department is NAC right now. The number of patients who received NACR was limited. We had more experiences to do surgery and postoperative care after NAC than NACR. The results need to be confirmed in multicenter randomized control trials in the future.

China has the highest incidence of ESCC worldwide $(27,28)$, and Henan Province contributes more than half of the number of cases in China (26). In our department, there were about 1,500 esophagectomies for cancer last year. That is an advantage to do clinical trials. Our aim was to explore the best preoperative combined treatment model for locally advanced resectable ESCC. The Academic Committee of $\mathrm{HCH}$ has already passed the randomized controlled trials protocol of $\mathrm{NAC} \pm \mathrm{NAR}$ versus $\mathrm{NACR} . \mathrm{NAC} \pm \mathrm{NAR}$ might deserve to be included in standard armamentarium for the treatment of locally advanced ESCC in the future.

\section{Conclusions}

In the present study, the NAC \pm NAR combined treatment model was tolerable. This model got the same survival benefits as NACR, avoids unnecessary chemoradiotherapy and achieved lower postoperative complication rates. In the future, it might be determined to be the best combined preoperative treatment for locally advanced ESCC, and for this reason, it deserves further exploration.

\section{Acknowledgements}

Funding: This project was supported by the Henan provincial public health authority (Grant number. 201501003), Henan province ministry of education (Grant number. 17A320048) and Wu Jieping Fund (Grant number. 320.6799.15062). Wu Jieping Fund (Grant number. 320.2730.1892).

\section{Footnote}

Conflicts of Interest: The authors have no conflicts of interest to declare.

Ethical Statement: The project was approved by the Review Board and Ethics Committee of Henan Cancer Hospital $(\mathrm{HCH}) /$ The affiliated Cancer Hospital of Zhengzhou
University. The ethical approval number is 2018122 .

\section{References}

1. Ando N, Kato H, Igaki H, et al. A randomized trial comparing postoperative adjuvant chemotherapy with cisplatin and 5 -fluorouracil versus preoperative chemotherapy for localized advanced squamous cell carcinoma of the thoracic esophagus (JCOG9907). Ann Surg Oncol 2012;19:68-74.

2. Ando N, Iizuka T, Ide H, et al. Surgery plus chemotherapy compared with surgery alone for localized squamous cell carcinoma of the thoracic esophagus: a Japan Clinical Oncology Group Study--JCOG9204. J Clin Oncol 2003;21:4592-6.

3. van Heijl M, van Lanschot JJ, Koppert LB, et al. Neoadjuvant chemoradiation followed by surgery versus surgery alone for patients with adenocarcinoma or squamous cell carcinoma of the esophagus (CROSS). BMC Surg 2008;8:21.

4. Sjoquist KM, Burmeister BH, Smithers BM, et al. Survival after neoadjuvant chemotherapy or chemoradiotherapy for resectable oesophageal carcinoma: an updated metaanalysis. Lancet Oncol 2011;12:681-92.

5. Kuroda J, Yoshida M, Kitajima M, et al. Utility of preoperative chemoradiotherapy for advanced esophageal carcinoma. J Gastroenterol Hepatol 2012;27 Suppl 3:88-94.

6. Mariette C, Dahan L, Mornex F, et al. Surgery Alone Versus Chemoradiotherapy Followed by Surgery for Stage I and II Esophageal Cancer: Final Analysis of Randomized Controlled Phase III Trial FFCD 9901. J Clin Oncol 2014;32:2416-22.

7. Kumagai K, Rouvelas I, Tsai JA, et al. Meta-analysis of postoperative morbidity and perioperative mortality in patients receiving neoadjuvant chemotherapy or chemoradiotherapy for resectable oesophageal and gastrooesophageal junctional cancers. Br J Surg 2014;101:321-38.

8. Nie L, Liu Y. Analysis of application of docetaxel combined therapy scheme in treating advanced head and neck neoplasm. Pak J Pharm Sci 2016;29:2231-4.

9. Yamasaki M, Yasuda T, Yano $M$, et al. Multicenter randomized phase II study of cisplatin and fluorouracil plus docetaxel (DCF) compared with cisplatin and fluorouracil plus Adriamycin (ACF) as preoperative chemotherapy for resectable esophageal squamous cell carcinoma (OGSG1003). Ann Oncol 2017;28:116-20.

10. Hara H, Tahara M, Daiko H, et al. Phase II feasibility study of preoperative chemotherapy with docetaxel, 
cisplatin, and fluorouracil for esophageal squamous cell carcinoma. Cancer Sci 2013;104:1455-60.

11. Eisenhauer EA, Therasse P, Bogaerts J, et al. New response evaluation criteria in solid tumours: revised RECIST guideline (version 1.1). Eur J Cancer 2009;45:228-47.

12. Zheng Y, Li Y, Liu X, et al. A phase III, multicenter randomized controlled trial of neo-adjuvant chemotherapy paclitaxel plus cisplatin versus surgery alone for stage IIAIIIB esophageal squamous cell carcinoma. J Thorac Dis 2017;9:200-4.

13. Zheng Y, Li Y, Wang Z, et al. A video demonstration of the Li's anastomosis-the key part of the "non-tube no fasting" fast track program for resectable esophageal carcinoma. J Thorac Dis 2015;7:1264-8.

14. Kaplan DK, Whyte RI, Donnelly RJ. Oesophagogastrectomy using stapling instruments. Eur J Cardiothorac Surg 1988;2:95-9.

15. Davies AR, Gossage JA, Zylstra J, et al. Tumor stage after neoadjuvant chemotherapy determines survival after surgery for adenocarcinoma of the esophagus and esophagogastric junction. J Clin Oncol 2014;32:2983-90.

16. Stahl M, Walz MK, Stuschke M, et al. Phase III comparison of preoperative chemotherapy compared with chemoradiotherapy in patients with locally advanced adenocarcinoma of the esophagogastric junction. J Clin Oncol 2009;27:851-6.

17. Burmeister BH, Thomas JM, Burmeister EA, et al. Is concurrent radiation therapy required in patients receiving preoperative chemotherapy for adenocarcinoma of the oesophagus? A randomised phase II trial. Eur J Cancer 2011;47:354-60.

18. Smithers BM, Thomson I. Neoadjuvant chemotherapy or chemoradiotherapy for locally advanced esophageal cancer. Thorac Surg Clin 2013;23:509-23.

19. Klevebro F, Alexandersson von Dobeln G, Wang N, et al. A randomized clinical trial of neoadjuvant chemotherapy versus neoadjuvant chemoradiotherapy for cancer of the oesophagus or gastro-oesophageal junction. Ann Oncol 2016;27:660-7.

20. Nakamura K, Kato K, Igaki H, et al. Three-arm phase III trial comparing cisplatin plus 5-FU (CF) versus docetaxel, cisplatin plus 5-FU (DCF) versus radiotherapy with $\mathrm{CF}$ (CF-RT) as preoperative therapy for locally advanced esophageal cancer (JCOG1109, NExT study). Jpn J Clin Oncol 2013;43:752-5.

21. Tang H, Tan L, Shen Y, et al. CMISG1701: a multicenter prospective randomized phase III clinical trial comparing neoadjuvant chemoradiotherapy to neoadjuvant chemotherapy followed by minimally invasive esophagectomy in patients with locally advanced resectable esophageal squamous cell carcinoma (c'T3-4aN0-1M0) (NCT03001596). BMC Cancer 2017;17:450.

22. Xi M, Liao Z, Deng W, et al. A Prognostic Scoring Model for the Utility of Induction Chemotherapy Prior to Neoadjuvant Chemoradiotherapy in Esophageal Cancer. J Thorac Oncol 2017;12:1001-10.

23. Zheng Y, Li Y, Liu X, et al. Reevaluation of Neoadjuvant Chemotherapy for Esophageal Squamous Cell Carcinoma: A Meta-Analysis of Randomized Controlled Trials Over the Past 20 Years. Medicine (Baltimore) 2015;94:e1102.

24. Somdyala NI, Bradshaw D, Gelderblom WC, et al. Cancer incidence in a rural population of South Africa, 1998-2002. Int J Cancer 2010;127:2420-9.

25. Lin $Y$, Totsuka $Y, H e ~ Y$, et al. Epidemiology of esophageal cancer in Japan and China. J Epidemiol 2013;23:233-42.

26. Chen $W$, Zheng R, Baade PD, et al. Cancer statistics in China, 2015. CA Cancer J Clin 2016;66:115-32.

27. Almhanna K, Shridhar R, Meredith KL. Neoadjuvant or adjuvant therapy for resectable esophageal cancer: is there a standard of care? Cancer Control 2013;20:89-96.

28. Siegel R, Desantis C, Jemal A. Colorectal cancer statistics, 2014. CA Cancer J Clin 2014;64:104-17.
Cite this article as: Zheng Y, Liu X, Zhang R, Wang Z, Sun H, Qin J, Liu S, Li Y. Neoadjuvant chemotherapy with or without neoadjuvant radiotherapy compared with neoadjuvant chemoradiotherapy for esophageal cancer. J Thorac Dis 2018;10(8):4715-4723. doi: 10.21037/jtd.2018.07.124 